EarthArxiv Preprint

The following unpublished, not yet peer-reviewed manuscript was submitted to the journal Open Quaternary on April 9, 2021.

You can download the teaching tool detailed in this manuscript: https://doi.org/10.5281/zenodo.4597618

Please feel free to contact the author with suggestions or tips: Bryan Lougheed Department of Earth Sciences, Uppsala University, Sweden bryan.lougheed@geo.uu.se

bonne journée 


\section{Orbital, the Box - An interactive educational tool for in-depth understanding of astronomical climate forcing.}

25 Bryan C. Lougheed

Department of Earth Sciences, Uppsala University, Uppsala, Sweden

bryan.lougheed@geo.uu.se

\section{Abstract}

30

“Orbital, the Box" provides an interactive tool with graphical user interface (GUI) for stimulating active, visual learning for understanding of astronomical climate forcing. This cross-platform tool can be run locally on a personal computer using a standard web browser environment with no need for plugins, thus maximising accessibility for students and teachers alike. The tool facilitates in the

35 development of a holistic and quantitative understanding of astronomical climate forcing by allowing students to independently vary orbital parameters, after which they can instantaneously see the resulting effect upon the seasonal and latitudinal distribution of solar irradiance arriving at the top of the Earth's atmosphere. Such an approach follows a classic controlled experimental design whereby one parameter can be changed while all others are kept constant. This experimental tool

40 can be deployed as a virtual laboratory, including within a flipped classroom setting, to promote active learning of traditionally challenging concepts such as the roles of eccentricity and precession in astronomical climate forcing, and in particular their interaction with Kepler's second law and the subsequent consequences for season length.

\section{$45 \quad 1.0$ Introduction}

Astronomical climate forcing is the theory that changes in a Earth's orbital configuration can influence its climate (Herschel, 1832; Adhémar, 1842; Croll, 1864). A major breakthrough in the furthering of this theory was achieved by the exhaustive calculations of the Serbian civil engineer

50 Milutin Milanković who, in the early $20^{\text {th }}$ century, used known orbital parameters to calculate secular changes in the seasonal and latitudinal distribution of incoming irradiance at the top of the Earth's atmosphere throughout the ages, and related these changes to the Quaternary ice ages. The decades of work is summed up in Milkanković's Kanon der Erdebestrahlung und seine Anwendung auf das Eiszeitenproblem (Milanković, 1941). Changes in the aforementioned seasonal and 
55 latitudinal distribution are an important driver of long-term climate changes on planet Earth and understanding thereof is a foundational element of geoscience education. However, quantitative teaching of the separate contributions of the various orbital parameters upon the irradiance distribution can often be a challenging task.

60 The three main parameters that can change the aforementioned seasonal and latitudinal distribution are obliquity of the ecliptic, eccentricity of the orbital ellipse and general precession (the combined effect of axial and apsidal precession), hereafter referred to simply as obliquity, eccentricity and precession. As outlined by Wampler (2000a), introductory teaching of astronomical climate forcing within geosciences should include the following:

65

(1) A description of the changes in physical motion caused by the parameters and some explanation about the causes.

(2) A description of the influence of the parameters upon the seasonal and latitudinal distribution of incoming irradiance.

Regarding the first point, teaching of astronomical climate forcing within geosciences curricula does impart a general explanation of the physical motion of the three main parameters (eccentricity, obliquity and precession) that is sufficient enough for purposes of palaeoclimate teaching. The

75 physical causes of the processes (i.e. gravitational interaction between the celestial bodies) are also touched upon.

The second point, a description of the influence of the parameters upon the seasonal and latitudinal distribution of incoming irradiance, is of great importance for understanding of palaeoclimate, and

80 is generally more difficult to teach in the case of some parameters, especially within a lecture and/or textbook format. In the case of obliquity, the influence upon the irradiance distribution is fortunately relatively straightforward to teach once the understanding is established that seasons on Earth are caused by obliquity in combination with the Earth's orbit around the Sun. From that base understanding, one can subsequently convey that greater obliquity will lead to greater seasonality 85 and vice-versa.

Teaching of the more nuanced changes caused by the combined influence of both eccentricity and precession can be challenging because it must necessarily invoke Kepler's second law, i.e. that the 
Earth will orbit at a faster speed when closer to the Sun and vice-versa. In my own experience, the

90 influence of these phenomena upon the Earth's climate are difficult to convey in a lecture setting, and exam questions relating to obliquity tend to be better answered than questions relating to eccentricity and precession, indicating a failure to fully convey these concepts. Communication with colleagues across multiple departments (Earth Sciences and Physics) confirmed that, in particular, the concept of precession consistently represents a "muddiest point” in multiple

95 disciplines. Due to the challenges in teaching this particular concept, and possibly also due to the fact that Keplerian concepts are difficult to convey graphically, textbooks traditionally touch on it only briefly. This has the potential for students developing incomplete understanding regarding astronomical climate forcing (Wampler, 2000b, 2000a). This incomplete understanding subsequently has the potential to persist into the scientific literature in some cases, as noted by

100 Bol'shakov (2017). Misrepresentations of Milanković's work in the literature include the claim that Milanković theory of astronomical forcing of glacial ablation/accumulation is based on highlatitude irradiance received on the day of the summer solstice (often erroneously referred to as “June 21” for all geological ages), whereas Milanković explicitly referred to not a single day, but the caloric summer half of the year, the sommerhalbjahr (Milanković, 1941). This type of

105 misrepresentation, in essence an oversimplification of Milanković theory, likely stems from from incomplete undergraduate teaching of the influence of the Keplerian orbit upon the Earth's season lengths and subsequent solar irradiance profile. Relatively recent studies in the literature have sought to comprehensively readdress such simplifications (Huybers, 2006, 2011; Berger et al., 2010), but these studies are not aimed at an undergraduate audience. Other misrepresentations can

110 include, e.g., orbital tuning of climate data to single orbital parameters (such as eccentricity) and citing Milanković as a justification, whereas Milanković theory pertains to the collective contribution of all orbital parameters to the distribution of incoming irradiance, and specifically with respect to Quaternary ice ages.

115 Interactive pedagogical method/tools can improve existing geoscience teaching methods, increasing attainment of understanding and student motivation when implemented within, e.g. a "flipped classroom” (Bykerk-Kauffman, 1995; Huguet et al., 2020). In the case of astronomical climate forcing, it is obviously not possible to apply the traditional interactive methods used within geosciences, e.g. a fieldwork or laboratory element. However, an interactive tool with graphical 120 user interface (GUI) can be developed to offer a virtual laboratory element (Kostadinov and Gilb, 2014). Here, Orbital, the Box is presented, which can be used to help imparting an understanding of orbital parameters upon the distribution of irradiance across latitudes and seasons, with the aim of 
developing both a conceptual (specifically, a sense of the physical mechanism) and quantitative understanding of processes. Quantitative understanding is particularly important and is often

125 overlooked within the geosciences (Manduca et al., 2008). Such a holistic and quantitative understanding of the effect of orbital parameters upon the Earth's irradiance profile is vital before the student can proceed on to other related subjects, such as learning about the millennial-scale time frequencies of past changes in eccentricity, obliquity and precession, and how they may or may not be manifested in the palaeoclimate record across vast timescales.

\subsection{Method}

\subsection{GUI interface}

135

The user is presented with a control panel in the form of three slider controls: (1) eccentricity, (2) obliquity and (3) $\omega$, the geocentric solar longitude $(\lambda)$ at which perihelion occurs, changes of which are caused by precessional processes. The user can set 15 unique values for eccentricity (covering a range of typical Earth values from 0.001 to 0.058 ), 15 unique values for obliquity (covering a range

140 of typical Earth values between $21.8^{\circ}$ and $24.5^{\circ}$ ) and 13 unique values for $\omega$ (from $0^{\circ}$ to $360^{\circ}$ ). Each time a slider is adjusted, the image to the right displaying the irradiance distribution and orbital information (distance from sun, orbital speed) is updated instantaneously, helping to facilitate rapid active learning. Each of the three controls can be set independently, meaning that there are a total of $15 \times 15 \times 13=2925$ possible unique combinations to be explored. The reason the

145 results can be loaded instantaneously on a standard personal computer is because the images containing the irradiance distributions are not computed live, instead, the respective images for all 2925 unique combinations have been pre-computed and can thus be instantly called upon when required. The control panel system used to call the images is written in HTML, CSS and Javascript, all of which are included with the major web browsers on all major computing platforms. As such,

150 the tool can be run on desktop computers, laptops or tablets. Publicly available tutorials were used to design the CSS and Javascript elements (w3schools.com, 2020).

\subsection{Computation of solar irradiance}

155 The 2925 unique irradiance scenarios were computed in Matlab 2019a using scripts based on established methodologies. These scripts were specifically written for the development of this 
educational tool, but could be used in other settings (e.g. research), and have therefore been made publicly available on Github (http://www.github.com/bryanlougheed/orbital_the_box/).

160 The image of annual irradiance distribution is calculated as follows. For each unique combination of eccentricity, obliquity and $\omega$, a $180^{\circ}$ latitude by 365.2 day grid of irradiance is produced, using a respective resolution of $0.5^{\circ}$ and 0.2 days. For the centre of each grid cell, $24 \mathrm{hr}$ mean irradiance (daily $\mathrm{Q}_{\text {mean }}$ ) is calculated in $\mathrm{Wm}^{-2}$ following Berger (1978), using a solar constant of $1361 \mathrm{Wm}^{-2}$. The aforementioned procedure requires $\lambda$ as input, and I have calculated the $\lambda$ associated with each

1650.2 day increment following the standard Keplerian methods outlined by Meeus (1998) involving a binary search solution for the Kepler equation developed by Sinnott (1985). To the best of my knowledge, this computational procedure for the Kepler equation was first highlighted within the geosciences by Kostadinov and Gilb (2014). The Berger (1978) irradiance procedure furthermore calls for geocentric latitude as input, but I have substituted it here by geographic latitude to account

170 for the ellipsoidal shape of the Earth (using the WGS84 reference ellipsoid). I validated this substitution approach against the incoming irradiance angle correction approach of Van Hemelrijck (1983). In order to better facilitate visual interpretation of the irradiance grid showing daily $\mathrm{Q}_{\text {mean }}$, it is contoured at $25 \mathrm{Wm}^{-2}$ intervals from 0 to $650 \mathrm{Wm}^{-2}$. The annual $\mathrm{Q}_{\text {mean }}$ for each latitude is calculated by taking the mean of all $24 \mathrm{hr} \mathrm{Q}_{\text {mean }}$ values calculated for each 0.2 day increment. Earth's

175 distance from the Sun for each $\lambda$ increment is calculated following the standard Keplerian methods outlined in Meeus (1998), assuming a semi-major axis of 1 AU. The corresponding orbital speed is calculated following the law of orbital energy invariance.

\subsection{Overview of the GUI tool}

\section{$180 \quad 3.1$ Graphical representation}

Within palaeoclimate teaching, current representations of the effect of orbital parameters upon the distribution of irradiance are sometimes not not described correctly (Wampler, 2000b, 2000a), possibly due to the limitations of the text format. Here, I have created a graphical representation of that captures many complex concepts in one glance, shown in Figure 1 for the orbital configuration

185 of the $21^{\text {st }}$ century (eccentricity: 0.0167 , obliquity: $23.4^{\circ}$, geocentric longitude of perihelion: $283^{\circ}$ ). This static graphical representation already allows a student to develop an understanding of the effects of eccentricity and precession upon the Earth's irradiance profile. For example, one sees that nowadays the Earth is closest to the Sun (i.e. at perihelion) approximately near the $280^{\text {th }}$ day of the tropical year, approximately coinciding with southern hemisphere summer. This means that southern hemisphere summer receives slightly higher peak irradiance than the northern hemisphere 
summer does, as is visualised in the figure. However, one can also see how the Earth's orbital speed is slightly faster during southern hemisphere summer, which translates to southern hemisphere summer being slightly shorter than northern hemisphere summer (as indicated by the day durations indicated in the figure). The season length compensates for the difference in irradiance received, meaning that any latitude will receive the same mean irradiance throughout the course of the entire tropical year as its corresponding latitude in the opposite hemisphere (as can be seen in the right panel).
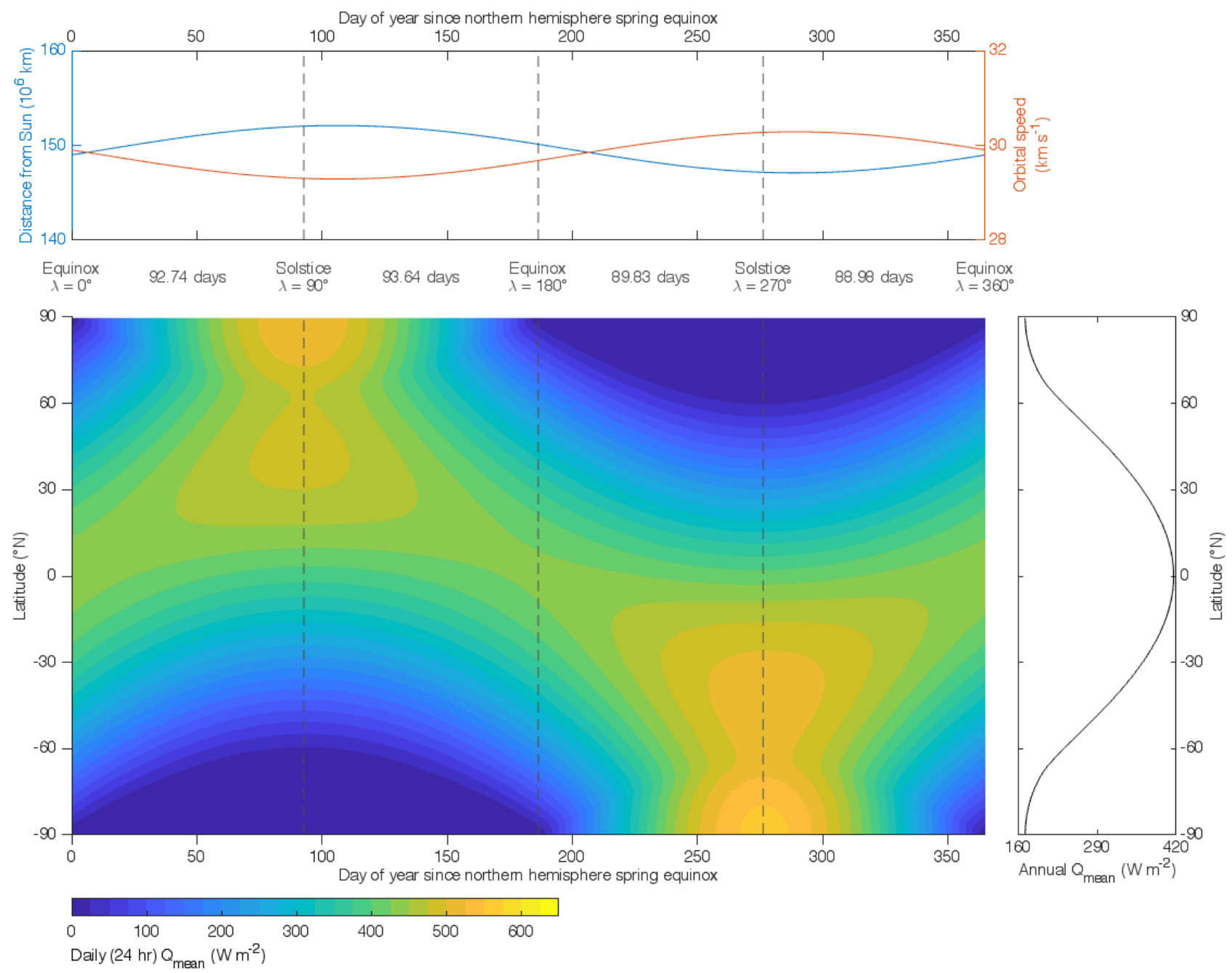

Figure 1. Graphical representation of the Earth's irradiance profile in the early $21^{\text {st }}$ century (eccentricity: 0.0167, obliquity: $23.4^{\circ}$, geocentric longitude of perihelion: $283^{\circ}$ ). Top panel: The Earth’s distance from the sun and the speed of its orbit throughout the tropical year, starting on the day of the spring equinox. Equinox and solstice days are indicated by dashed lines. Bottom-left panel: Distribution of irradiance (24-hour mean irradiance in $\mathrm{Wm}^{-2}$ ) for the latitudes of Earth throughout the tropical year, also starting on the day of the spring equinox. Equinox and solstice days are indicated by dashed lines. Bottom-right panel: The mean irradiance received at each latitude throughout the course of the entire tropical year. 


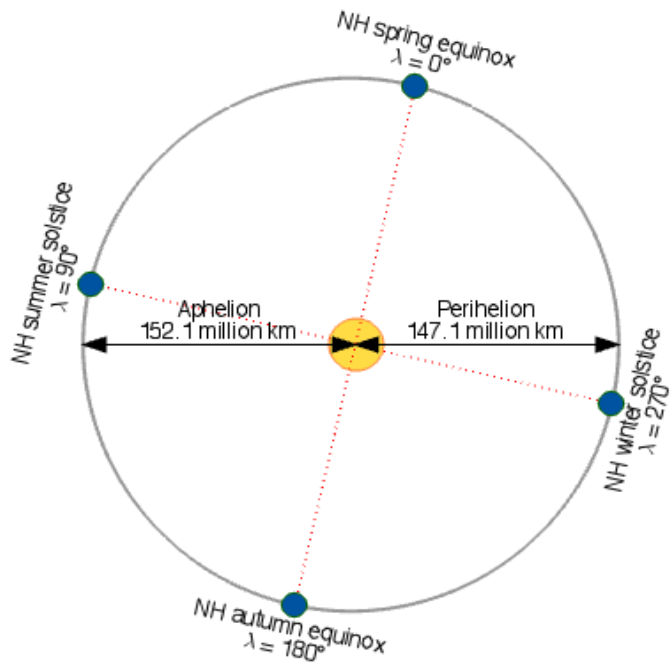

Figure 2. Graphical representation of the Earth's orbital configuration in the early $21^{\text {st }}$ century (eccentricity: 0.0167, geocentric longitude of perihelion: $283^{\circ}$ ). The orbital shape has been drawn to scale. The size of the Earth and Sun have been increased for visual clarity.

In addition to the graphical representation of the irradiance distribution reaching the Earth, a scale drawing of the elliptical orbit of the Earth, with the Sun at the focal point, is also provided (Figure 2). This figure allows the student to visually analyse the aphelion and perihelion distances, and how the tropical year is oriented with regards to the orbit due to general precession.

\subsection{Interactive representation}

The graphical representations shown in Figure 1 and Figure 2 are a static representation of a single orbital configuration corresponding to the orbital parameters of the early $21^{\text {st }}$ century. Here, this

225 graphical representation is used to form the basis of an interactive tool. It has previously been demonstrated that such interactive tools with real-time updated results help students to more thoroughly build a conceptual understanding of a complex process, especially when the student is allowed to independently explore the possibilities provided by the tool (Wieman et al., 2008; National Research Council, 2015). In the case of "Orbital, the Box”, the student can experiment by using a control panel (Figure 3) to independently change orbital parameters and see how such changes influence the following: (1) the variation in Earth's orbital speed and distance from the Sun throughout the year; (2) the irradiance distribution across the Earth's latitudes and throughout the year; (3) the day length of the astronomical seasons; (4) the mean annual irradiance received at each latitude; (5) the shape and positioning of the Earth's orbit relative to the Sun. This setup constitutes

235 a classic experimental environment with multiple variables (in this case three), whereby one variable is changed while the other two are kept constant. 
Figure 3. The slider control panels used to control the graphical representations.

\subsection{Digital textbook}

Included in the interactive GUI is access to a very basic digital textbook explaining the concepts of obliquity, eccentricity and geocentric longitude of perihelion (which is governed by precession). The hyperlinks above the slider controls in Figure 3 will take the students to pages with basic information about the orbital parameters and a tutorial containing example calculations and figures demonstrating basic principles. In the obliquity section, students learn how to calculate the declination latitude of the Sun, and the midday angle of the Sun for the solstices at their city, given a specific obliquity. In the eccentricity section, there is a tutorial on how to calculate aphelion and perihelion distances given a specific eccentricity, as well as information on how to calculate the

250 speed of the Earth’s orbit at aphelion and perihelion. Geocentric longitude of perihelion is explained and its cause (general precession) is explained using animations.

\subsection{Conclusion and reuse potential}

255 The GUI tool has great potential to be integrated into geosciences curricula, particularly within a classic experimental environment allowing for the isolated study of the individual effects of orbital parameters upon the seasonal and latitudinal distribution of irradiance. The tool provides an experimental environment that can be deployed within a flipped classroom setting with jigsaw activities, as has previously been successfully deployed in geosciences the case of, e.g. plate tectonics (Sawyer et al., 2005). An example lesson plan is to split students into three groups, with each group focussing on learning the effect of one (or all) of the three orbital parameters (obliquity, eccentricity and precession). Each group could investigate the effect (if any) of their parameter(s) upon orbital distance, speed, seasonal irradiance distribution, length of season, and mean annual irradiance received at each latitude. Afterwards, groups can rotate members and/or hold discussions to teach each the other groups about the particular orbital parameter that they studied. 
It should be noted that the GUI tool developed here exclusively includes realistic values for the orbital parameters of planet Earth. A useful additional exercise/challenge is assigning groups unrealistic parameters for Earth (such as an obliquity value of $45^{\circ}$ ) and asking them to then sketch

270 the irradiance profile that they would expect to be associated with such a parameter, as well as what latitude the polar circle and tropics would be. Such a task can promote metacognitive thinking, as it challenges the students to extrapolate an extreme hypothetical scenario, which can in turn help them better understand the functioning of more realistic scenarios.

275 Planet Earth's irradiance distribution and its relation to orbital parameters is a very challenging subject to teach. Geosciences has traditionally relied upon fieldwork and/or laboratory settings to stimulate active learning, but carrying out real-world experiments upon the Earth's orbit is beyond current technology and probably undesirable. A computer-based experimental environment can allow for similar interactive attainment of understanding as would be attained in a real-world experimental environment.

\section{Software availability}

The Orbital, the Box interactive teaching tool can be downloaded from Zenodo: https://doi.org/10.5281/zenodo.4597618. Matlab scripts that can be used to recreate the irradiance and orbital figures shown in the tool can be downloaded from Github: https://github.com/bryanlougheed/orbital the box

\section{Acknowledgements}

290 The author acknowledges Swedish Research Council (Vetenskapsrådet - VR) Starting Grant number 2018-04992. Yunus Baykal, Jorijntje Henderiks, Boris-Theofanis Karatsolis, Patrik Thunström, Niklas Wahlström (all Uppsala University) and Brett Metcalfe (Wageningen UR \& Vrije Universiteit Amsterdam) are thanked for discussions.

\section{References}

Adhémar, J. A.: Révolutions de la mer, déluges périodiques, Carilian-Goeury et V. Dalmont, Paris., 1842. 
Berger, A., Loutre, M.-F. and Yin, Q.: Total irradiation during any time interval of the year using elliptic integrals, Quaternary Science Reviews, 29(17), 1968-1982, doi:10.1016/j.quascirev.2010.05.007, 2010.

Berger, A. L.: Long-Term Variations of Daily Insolation and Quaternary Climatic Changes, J. Atmos. Sci., 35(12), 2362-2367, doi:10.1175/1520-0469(1978)035<2362:LTVODI>2.0.CO;2, 1978.

Bol'shakov, V. A.: On the ill-defined notion of the Milankovitch Theory and its influence on the development of the orbital theory of the paleoclimate, Her. Russ. Acad. Sci., 87(4), 356-369, doi:10.1134/S1019331617040025, 2017.

Bykerk-Kauffman, A.: Using Cooperative Learning in College Geology Classes, Journal of Geological Education, 43(4), 309-316, doi:10.5408/0022-1368-43.4.309, 1995.

Croll, J.: XIII. On the physical cause of the change of climate during geological epochs, The London, Edinburgh, and Dublin Philosophical Magazine and Journal of Science, 28(187), 121-137, 1864.

Herschel, J. F. W.: XVII.-On the Astronomical Causes which may influence Geological Phænomena., Transactions of the Geological Society of London, S2-3(2), 293-300, doi:10.1144/transgslb.3.2.293, 1832.

Huguet, C., Pearse, J., Noè, L. F., Valencia, D. M., Ruiz, N. C., Heredia, A. J. and Avedaño, M. A. P.: Improving the motivation of students in a large introductory geoscience course through active learning, Journal of Geoscience Education, 68(1), 20-32, doi:10.1080/10899995.2019.1588489, 2020.

Huybers, P.: Early Pleistocene Glacial Cycles and the Integrated Summer Insolation Forcing, Science, 313(5786), 508-511, doi:10.1126/science.1125249, 2006.

Huybers, P.: Combined obliquity and precession pacing of late Pleistocene deglaciations, Nature, 480(7376), 229-232, doi:10.1038/nature10626, 2011.

Kostadinov, T. S. and Gilb, R.: Earth Orbit v2.1: a 3-D visualization and analysis model of Earth's orbit, Milankovitch cycles and insolation, Geosci. Model Dev., 7(3), 1051-1068, doi:10.5194/gmd7-1051-2014, 2014. 
Manduca, C. A., Baer, E., Hancock, G., Macdonald, R. H., Patterson, S., Savina, M. and Wenner, J.: Making Undergraduate Geoscience Quantitative, Eos Trans. AGU, 89(16), 149, doi:10.1029/2008EO160001, 2008.

Meeus, J.: Astronomical Algorithms, 2nd ed., Willmann-Bell, Inc., Richmond, Virginia., 1998.

Milanković, M.: Kanon der Erdebestrahlung und seine Anwendung auf das Eiszeitenproblem, Königlich Serbische Akademie., 1941.

National Research Council: Reaching Students: What Research Says About Effective Instruction in Undergraduate Science and Engineering, National Academies Press, Washington, D.C., 2015.

Sawyer, D. S., Henning, A. T., Shipp, S. and Dunbar, R. W.: A Data Rich Exercise for Discovering Plate Boundary Processes, Journal of Geoscience Education, 53(1), 65-74, doi:10.5408/1089-999553.1.65, 2005.

Sinnott, R. W.: A computer assault on Kepler’s equation, Sky \& Telescope, 70, 158-159, 1985.

Van Hemelrijck, E.: The oblateness effect on the extraterrestrial solar radiation, Solar Energy, 31(2), 223-228, doi:10.1016/0038-092X(83)90085-3, 1983.

Wampler, J. M.: Misconceptions - A Column about Errors in Geoscience Textbooks: Confusion about the Changing Eccentricity of the Earth's Orbit, Journal of Geoscience Education, 48(4), 541543, doi:10.5408/1089-9995-48.4.541, 2000a.

Wampler, J. M.: Misconceptions - A Column about Errors in Geoscience Textbooks:

Misconceptions of Changes in Obliquity of the Ecliptic, Journal of Geoscience Education, 48(5), 701-704, doi:10.5408/1089-9995-48.5.701, 2000b.

Wieman, C. E., Adams, W. K. and Perkins, K. K.: PhET: Simulations That Enhance Learning, Science, 322(5902), 682-683, doi:10.1126/science.1161948, 2008.

w3schools.com. How To - Range Sliders. Available at https://www.w3schools.com/howto/howto_js_rangeslider.asp [last accessed 28 December 2020]. 